\title{
COHOMOLOGY RING OF THE ORBIT SPACE OF CERTAIN FREE $Z_{p}$-ACTIONS
}

\author{
RONALD M. DOTZEL AND TEJ B. SINGH
}

(Communicated by Thomas Goodwillie)

\begin{abstract}
In this paper, we consider actions of $G=Z_{p}$ (with $p$ an odd prime) on spaces $X$ which are of cohomology type $(0,0)$ (i.e., have the mod- $p$ cohomology of the one-point union of an $n$-sphere, a $2 n$-sphere and a a $3 n$-sphere, $n$ odd). If $X$ is not totally non-homologous to zero in $X_{G}$ we determine the fixed set, give examples of all possibilities for the fixed set and compute the cohomology ring structure of the orbit space in the case where $G$ acts freely. In [4], we considered fixed sets for related spaces, when $X$ is totally non-homologous to zero in $X_{G}$.
\end{abstract}

\section{INTRODUCTION}

Let $X$ be a finite $\mathrm{CW}$ complex with cohomology groups satisfying:

$$
H^{j}(X ; Z)= \begin{cases}Z, & j=0, n, 2 n, 3 n, \\ 0, & \text { otherwise. }\end{cases}
$$

If $u_{i}$ generates $H^{i n}(X ; Z), i=1,2,3$, we say that $X$ has cohomology type $(a, b)$ when $u_{1}^{2}=a u_{2}$ and $u_{1} u_{2}=b u_{3}$ (terminology due to Toda [7]). Let $G=$ $Z_{p}\left(p\right.$ an odd prime) act on $X$. If $b \neq 0(\bmod p)$, then either $X \simeq_{p} S^{n} \times S^{2 n}$ or $X \simeq_{p} P^{3}(n)$ depending on whether $a=0(\bmod p)$ or $a \neq 0(\bmod p)$. Here $X \simeq_{p} Y$ means that $X$ and $Y$ have isomorphic mod- $p$ cchomology rings. When $b \neq 0(\bmod p)$ the nature of the fixed set of $G$ on $X$ has been studied in detail ([5], [6], [8]). In [4] we considered the case $b=0(\bmod p)$, when $X$ is totally non-homologous to zero in $X_{G}(\bmod p)$. The structure of the possible fixed sets was determined, and it was noted that when $n$ is even, $X$ is always totally non-homologous to zero. Here we settle the remaining case where $X$ is not totally non-homologous to zero $(\bmod p)$, so that $n$ is necessarily odd. Since $n$ is odd, we must have $a=0(\bmod p)$ and $X$ is of cohomology type $(0,0)$. We obtain

Theorem 1. Let $G=Z_{p}, p$ an odd prime, act on a finite complex $X$ of cohomology type $(0,0)(\bmod p)$. If $X$ is not totally non-homologous to zero in $X_{G}$, then the fixed point set $F \simeq_{p} S^{q},-1 \leq q \leq 3 n, q$ odd. Moreover, all possibilities for $q$ occur.

Received by the editors December 1, 1992 and, in revised form, May 26, 1994.

1991 Mathematics Subject Classification. Primary 55S17; Secondary 55R20. 
Examples of $G$ acting freely on spaces $X$ of type $(0,0)$ were constructed in [4]. Here we compute the cohomology of the orbit space of a free $G$ action on $X$ obtaining

Theorem 2. Let $X$ be a space of cohomology type $(0,0)$ with nonzero mod-p cohomology only in dimensions $0, n, 2 n, 3 n$ ( $n$ odd). Suppose $G=Z_{p}, p$ odd prime, acts freely on $X$. Then as graded commutative algebras,

$$
H^{*}\left(X / G ; Z_{p}\right)=Z_{p}[x, y, z] /\left(x^{2}, z^{2}, z y^{n+1 / 2}, y^{3 n+1 / 2}\right)
$$

where $\operatorname{deg} x=1, \operatorname{deg} y=2, \operatorname{deg} z=n$ and $y=\beta_{p}(x) \quad\left(\beta_{p}\right.$ being the mod- $p$ Bockstein).

\section{Preliminaries}

We will recall here several facts about equivariant cohomology $H_{G}^{*}(X)=$ $H^{*}\left(X_{G}\right)$ (see [2, Chapter 7] for more information).

First of all we will denote cohomology with $Z_{p}$ coefficients simply by $H^{*}(X)$ and from now on $Z_{p}$ coefficients are intended (unless explicitly indicated otherwise). If $G=Z_{p}$ acts on $X, F$ denotes the fixed set. It is well known that if $H^{*}(X)=0$ for $^{*}>m$, then the inclusion $F_{G} \rightarrow X_{G}$ induces a cohomology isomorphism $H^{*}\left(X_{G}\right) \rightarrow H^{*}\left(F_{G}\right)$ for ${ }^{*}>m$. Recall that $X_{G}=E G \times_{G} X$ is the bundle over the classifying space $B G$ with fibre $X$ associated to the principal bundle $E G \rightarrow B G \cdot F_{G}=F \times B G$ is a subbundle.

If $X$ is a finite $G$-CW-complex and $G$ acts trivially on $H^{*}(X ; Z)$ (integer coefficients), then for any $k$,

$$
\sum_{i \geq 0} \mathrm{rk} H^{k+2 i}(F) \leq \sum_{i \geq 0} \mathrm{rk} H^{k+2 i}(X) .
$$

For example, if $H^{*}(X)$ vanishes in even degrees, so does $H^{*}(F)$.

We recall that if $\sum$ rk $H^{*}(X)<\infty$ (as in the present case), then $X$ is totally non-homologous to zero in $X_{G}$ (i.e., there is a cohomology extension of the fibre $\left.X \rightarrow X_{G}\right)$ with $Z_{p}$-coefficients iff $\sum \operatorname{rk} H^{*}(F)=\sum \operatorname{rk} H^{*}(X)$ iff $G$ acts trivially on $H^{*}(X)$ and the Leray-Serre spectral sequence of $X^{i} \rightarrow X_{G} \stackrel{\pi}{\rightarrow} B G$ degenerates.

In computing the cohomology of the orbit space $X / G$, where $G$ acts freely on $X$, we make use of the Leray-Serre spectral sequence of $X_{G} \rightarrow B G$ (in this case the map of orbit spaces $X_{G} \rightarrow X / G$ is a homotopy equivalence, so the cohomology of $X_{G}$ obtained from this spectral sequence will be the cohomology of $X / G)$. If $\pi_{1}(B G)=Z_{p}$ acts trivially on $H^{*}(X)$ (as it does in the present case), then the $E_{2}$ term is $E_{2}^{k, l}=H^{k}(B G) \otimes H^{l}(X)$. The product structure in the spectral sequence induces a product in the subalgebras $E_{2}^{*, 0}$ and $E_{2}^{0, *}$ which coincides with the cup products. Also the edge homomorphisms,

$$
\begin{gathered}
H^{k}(B G)=E_{2}^{k, 0} \rightarrow E_{3}^{k, 0} \rightarrow \cdots \rightarrow E_{k+1}^{k, 0}=E_{\infty}^{k, 0} \subseteq H^{k}\left(X_{G}\right), \\
H^{l}\left(X_{G}\right) \rightarrow E_{\infty}^{0, l}=E_{l+1}^{0, l} \subset \cdots \subset E_{2}^{0, l}=H^{l}(X)
\end{gathered}
$$

are the homomorphisms $\pi^{*}: H^{k}(B G) \rightarrow H^{k}\left(X_{G}\right)$ and $i^{*}: H^{l}\left(X_{G}\right) \rightarrow H^{l}(X)$, respectively. 
Finally recall that

$$
H^{*}(B G)=Z_{p}[s, t] /\left(s^{2}\right)=\Lambda(s) \otimes Z_{p}[t]
$$

where $\operatorname{deg} s=1, \operatorname{deg} t=2$ and $\beta_{p}(s)=t \quad\left(\beta_{p}\right.$ is the mod-p Bockstein associated to $Z_{p} \rightarrow Z_{p^{2}} \rightarrow Z_{p}$ ).

\section{Proof of Theorem 1 AND EXAMPLeS}

To prove Theorem 1, suppose that $X$ is not totally non-homologous to zero in $X_{G}(\bmod p)$. Then we must have $n$ odd $\left(\right.$ by [4]) and $\operatorname{rk} H^{*}(F)<\operatorname{rk} H^{*}(X)=$ 4. Also $\chi(F) \equiv \chi(X) \equiv 0(\bmod p)$. So $\chi(F)=0$ or $\chi(F)=3$ and $p=$ 3. But $\chi(F)=3$ requires $\sum$ rk $H^{2 i}(F)>2$ and, since $G$ acts trivially on $H^{*}(X ; Z)$ (integer coefficients), we must have

$$
\sum \mathrm{rk} H^{2 i}(F) \leq \sum \mathrm{rk} H^{2 i}(X)=2 \text {. }
$$

Therefore $\chi(F)=0$ and $F \simeq_{p} S^{q}$ for $q$ odd and $-1 \leq q \leq 3 n$.

We now will give examples to show that all the possibilities for $q$ are actually realised. The case of $q=-1$ (i.e., $F=\varnothing$ ) is [4]. So assume that $q$ is odd $1 \leq q$. To begin with, let $n \geq 3$ be odd and $m=n+2$ (so $m$ is odd). There is a $Z_{p}$ action on $S^{2} \times S^{m}$ with fixed set $S^{3}$. One can obtain such an action by letting $\eta$ be the Hopf 2 -plane bundle over $S^{2},-\eta$ its inverse (i.e., $\eta \oplus-\eta=$ trivial 4-plane bundle). Let $\varepsilon$ be a trivial $m-3$ plane bundle. $-\eta \oplus \varepsilon$ admits a fibre-wise orthogonal action of $Z_{p}$ which leaves only the zero section (i.e., $S^{2}$ ) fixed. Consider $\eta \oplus(-\eta \oplus \varepsilon)$. Let $Z_{p}$ act trivially on $\eta$. Taking unit sphere bundles yields an action of $Z_{p}$ on $S^{2} \times S^{m}(m=n+2)$ with fixed set the total space of the sphere bundle of $\eta$ (i.e., $S^{3}$ ) (see [3] for other such examples). Now remove a fixed point to obtain a $Z_{p}$ action on a space $Y$ which is homotopy equivalent to $S^{2} \vee S^{n+2}$ and has contractible fixed set. Let $Z_{p}$ act trivially on $S^{n-3}$, and take the join of $S^{n-3}$ and $Y$. This space $W$ has $Z_{p}$ action with contractible fixed set and is itself homotopy equivalent to $S^{n} \vee S^{2 n}$. Now let $Z_{p}$ act on $S^{3 n}$ with fixed set $S^{q}$ for $q$ odd (e.g., take a linear action) and form the one-point union (at a fixed point) of $W$ and $S^{3 n}$. This provides all examples.

\section{Proof of Theorem 2}

By the Universal Coefficient Theorem, we have $H^{i n}(X)=Z_{p}$ for $i=$ $0,1,2,3$. We choose generators $v_{i} \in H^{i n}(X), i=1,2,3$, respectively, satisfying the relations $v_{1}^{2}=0$ and $v_{1} v_{2}=0$. Consider the Leray-Serre spectral sequence of the map $\pi: X_{G} \rightarrow B G$ with coefficients in the constant sheaf $\mathscr{H}^{*}(X)$ associated to $G=Z_{p}\left(G=Z_{p}=\pi_{1}(B G)\right.$ acts trivially on $\left.H^{*}(X)\right)$. The $E_{2}$-term of the spectral sequence is

$$
E_{2}^{k, l}=H^{k}(B G) \otimes H^{l}(X) .
$$

Since $X$ has no fixed points and $p$ is odd, $n$ must be odd and $E_{2} \neq E_{\infty}$. So some differential:

$$
d_{r}: E_{r}^{k, l} \rightarrow E_{r}^{k+r, l-r+1}
$$

must be nontrivial. This is only possible for $r=n+1,2 n+1$ and $3 n+1$, and it is easily seen that

$$
d_{n+1}\left(1 \otimes v_{1}\right)=d_{n+1}\left(1 \otimes v_{3}\right)=0 \quad \text { and } \quad d_{n+1}\left(1 \otimes v_{2}\right) \neq 0 .
$$


So

$$
E_{r}^{k, 2 n}=0=E_{r}^{k+n+1, n}
$$

for all $k$ and $r>n+1$. Obviously then, $d_{2 n+1}=0$.

If $d_{3 n+1}\left(1 \otimes v_{3}\right)=0$, then the bottom and top lines of the spectral sequence survive to infinity and this contradicts the fact that $H^{*}\left(X_{G}\right)=0$ for ${ }^{*}>3 n$ (for $G$ acts freely, hence $F_{G}=\varnothing$ ). Therefore $d_{3 n+1}\left(1 \otimes v_{3}\right) \neq 0$ so that

$$
E_{\infty}^{k, 3 n}=0=E_{\infty}^{k+3 n+1,0} \text {. }
$$

Hence we obtain

$$
H^{j}\left(X_{G}\right)= \begin{cases}Z_{p} & \text { for } j>3 n \\ Z_{p} & \text { for } 0 \leq j \leq n-1 \text { and } 2 n+1 \leq j \leq 3 n \\ Z_{p} \oplus Z_{p} & \text { for } n \leq j \leq 2 n\end{cases}
$$

To determine the multiplicative structure, note that for $k \leq 3 n, E_{2}^{k, 0} \subset$ $H^{k}\left(X_{G}\right)$. Let $x=s \otimes 1 \in E_{\infty}^{1,0}$ and $y=t \otimes 1 \in E_{\infty}^{2,0}$. Then $\pi^{*}(s)=x$, $\pi^{*}(t)=y$ and $\beta_{p}(x)=y$, by naturality of the Bockstein cohomology operation. The homomorphism

$$
y \cup(\cdot): E_{\infty}^{k, l} \rightarrow E_{\infty}^{k+2, l}
$$

is an isomorphism for $k \leq 3 n-2$ if $l=0$ and for $k \leq n-2$ if $l=n$. Therefore multiplication by $y \in H^{2}\left(X_{G}\right)$

$$
y \cup(\cdot): H^{k}\left(X_{G}\right) \rightarrow H^{k+2}\left(X_{G}\right)
$$

is an isomorphism for $k \leq 2(n-1)$. The element $1 \otimes v_{1} \in E_{2}^{0, n}$ is a permanent cocycle and determines an element $w$ in $E_{\infty}^{0, n} \cdot E_{2}^{*, *}$, and $E_{\infty}^{*, *}$ are bigraded commutative algebras and therefore the total complex Tot $E_{\infty}^{*, *}$ given by

$$
\left(\text { Tot } E_{\infty}^{*, *}\right)^{m}=\bigoplus_{k+l=m} E_{\infty}^{k, l}
$$

is a graded commutative algebra isomorphic to

$$
Z_{p}[x, y, w] /\left(x^{2}, w^{2}, w y^{(n+1) / 2}, y^{(3 n+1) / 2}\right) .
$$

Now we choose an element $z \in H^{n}\left(X_{G}\right)$ such that $i^{*}(z)=v_{1}$. Because the composition $\pi i$ factors through a point, $i^{*} \pi^{*}$ is zero in positive degrees and hence we can assume that $z y^{(n+1) / / 2}=0$. Since the multiplication by $y$ is an isomorphism in degrees less than $2(n-1), z y^{i} \neq 0$ for $2 i<n-1$. Thus we have

$$
H^{*}\left(X_{G}\right)=Z_{p}[x, y, z] /\left(x^{2}, z^{2}, z y^{(n+1) / 2}, y^{3 n+1) / 2}\right)
$$

as graded commutative algebras. $\pi: X_{G} \rightarrow X / G$ is a homotopy equivalence and so induces a cohomology isomorphism. This completes the proof.

\section{REFERENCES}

1. A. Borel et al., Seminar on transformation groups, Ann. of Math Stud., no. 46, Princeton Univ. Press, Princeton, NJ, 1960.

2. G. Bredon, Inroduction to compact transformation groups, Academic Press, New York, 1972.

3. Cohomological aspects of transformation groups, Proceedings of the Conference on Transformation Groups (New Orleans, 1967), Springer-Verlag, Berlin and New York, 1968, pp. 245-280. 
4. R. Dotzel and T. Singh, $Z_{p}$ actions on spaces of cohomology type $(a, 0)$, Proc. Amer. Math. Soc. 113 (1991), 875-878.

5. J. C. Su, Periodic transformations on the product of two spheres, Trans. Amer. Math. Soc. 112 (1964), 369-380.

6. _ Transformation groups on cohomology projective spaces, Trans. Amer. Math. Soc. 106 (1963), 305-318.

7. H. Toda, Note on cohomology ring of certain spaces, Proc. Amer. Math. Soc. 14 (1963), 89-95.

8. P. Tomter, Transformation groups on cohomology product of spheres, Invent. Math. 23 (1974), 79-88.

Department of Mathematics and Computer Science, University of Missouri-St. Louis, ST. LOUIS, MISSOURI 63121

Department of Mathematics, University of Delhi, Delhi-1 10 007, INDia 